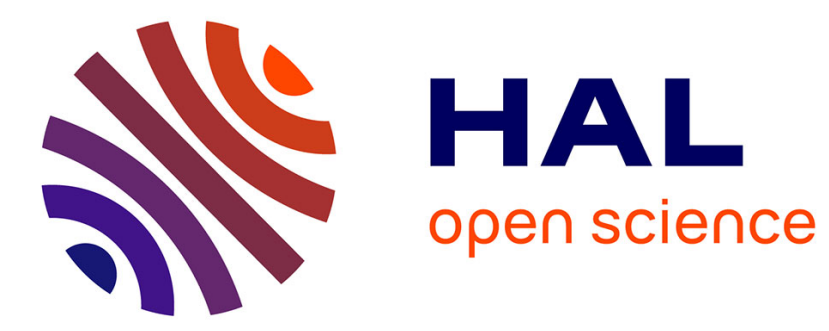

\title{
Renégotiation de contrats dans l'industrie du transport urbain en France
}

Philippe Gagnepain, Marc Ivaldi, David Martimort

\section{To cite this version:}

Philippe Gagnepain, Marc Ivaldi, David Martimort. Renégotiation de contrats dans l'industrie du transport urbain en France. Revue Economique, 2009, 60, pp.927-947. hal-00622833

\section{HAL Id: hal-00622833 \\ https://hal.science/hal-00622833}

Submitted on 13 Sep 2011

HAL is a multi-disciplinary open access archive for the deposit and dissemination of scientific research documents, whether they are published or not. The documents may come from teaching and research institutions in France or abroad, or from public or private research centers.
L'archive ouverte pluridisciplinaire HAL, est destinée au dépôt et à la diffusion de documents scientifiques de niveau recherche, publiés ou non, émanant des établissements d'enseignement et de recherche français ou étrangers, des laboratoires publics ou privés. 


\title{
Renégociation de contrats dans l'industrie du transport urbain en France ${ }^{1}$
}

\author{
Philippe Gagnepain, ${ }^{2}$ Marc Ivaldi et David Martimort ${ }^{3}$
}

\begin{abstract}
Résumé
Cet article analyse la renégociation des contrats de gestion déléguée dans le secteur du transport en France. Nous décrivons tout d'abord brièvement le secteur, puis nous présentons dans ses grandes lignes un modèle théorique de renégociation des contrats expliquant les évolutions dynamiques des subventions en direction des opérateurs et notamment leur profil croissant au cours du temps. Ce modèle est ensuite estimé. L'analyse économétrique montre que l'hypothèse consistant à supposer que les autorités ont une forte capacité d'engagement conduit à surestimer l'efficacité des firmes du secteur.
\end{abstract}

\begin{abstract}
The renegotiation of contracts in the French urban transport industry. This paper describes the renegotiation of delegated management contracts in the French urban transport. We briefly describe the sector, sketch a theoretical model showing that contract renegotiation explains an increasing profile of
\end{abstract}

\footnotetext{
${ }^{1}$ Les trois auteurs sont membres du CEPR. Les auteurs remercient pour leurs commentaires constructifs un rapporteur pour cette revue, les participants au Congrès 2007 de l'Association Française d'Economie, Paris Sorbonne, ainsi que le Centre d'Etude et de Recherche sur le Transport Urbain (CERTU, Lyon, France). Gagnepain remercie Ministerio de Educación y Ciencia (SEJ2004-00670 et SEJ2007-66268), de même que Fundación BBVA pour leur assistance financière. Martimort et Ivaldi remercient l'Agence Nationale pour la Recherche pour son soutien financier.

${ }^{2}$ Universidad Carlos III de Madrid; Departamento de Economía; C/ Madrid 126; 28903 Getafe (SPAIN).philippe@eco.uc3m.es.

${ }^{3}$ Toulouse School of Economics; Manufacture des Tabacs; 21, Allée de Brienne; 31000 Toulouse (France). ivaldi@cict.fr. martimor@cict.fr.
} 
subsidies over time. This model is finally estimated. Our analysis shows that wrongly assuming that local governments can fully commit to a profile of subsidies and do not renegotiate contracts leads to systematically overestimating the efficiency of firms in the sector.

JEL Classification: H11, D82.

\section{Introduction}

L'analyse des relations contractuelles entre collectivités locales soucieuses de trouver les meilleures conditions pour la fourniture des grands services publics ou environnementaux et le secteur privé est un sujet de la plus haute importance. Quels sont les modes contractuels qui préservent au mieux les intérêts de la collectivité? Quelles frictions peuvent éventuellement limiter l'efficacité de la gestion déléguée de ces grands services au secteur privé? L'économiste peut-il quantifier ces frictions et éventuellement fournir quelques principes de politiques économiques qui seraient autant de guides pour le décideur public?

Au moment de répondre à ces interrogations, on ne peut nier les tentations idéologiques qui consistent le plus souvent à arguer de manière polémique qui en faveur d'un mode administré de ces services, qui en faveur du seul secteur privé. Force est cependant de reconnaître qu'il existe encore un fossé important entre la sophistication des modèles que le théoricien a développé à ce jour pour décrire la relation contractuelle entre déléguant et délégataire ${ }^{4}$ et les leçons pratiques que l'on peut en retirer dès lors que le praticien ou le décideur public s'intéresse au bon fonctionnement d'un secteur donné.

D'un point de vue général, cet article a pour modeste objectif de contribuer à combler ce vide et de démontrer qu'en la matière certaines des interrogations que nous évoquions cidessus peuvent être levées. Prenant comme objet d'analyse l'industrie du transport urbain en France, nous proposons un modèle théorique et une application empirique expliquant les choix de contrats et de régulation des coûts des opérateurs. Notre analyse souligne que ces choix sont dictés d'une part par l'asymétrie d'information entre autorités publiques et opérateurs privés mais aussi par la capacité d'engagement parfois limitée des autorités publiques. Information asymétrique et engagement limité constituent autant de frictions contractuelles essentielles dont l'étude est indispensable si l'on veut évaluer l'efficacité des

\footnotetext{
${ }^{4}$ Voir par exemple Laffont et Tirole [1993] et Laffont et Martimort [2002].
} 
modes opératoires dans le secteur.

Dans chaque zone urbaine, le service de transport urbain est assuré par un opérateur unique et est réglementé par une autorité publique locale. Ces deux parties sont liées par un contrat qui est en pratique de type «coût du service» ou «prix fixe». ${ }^{5}$ L'autorité publique n'est capable ni d'observer l'efficacité technologique de l'opérateur ni d'évaluer les efforts de réduction des coûts que ce dernier peut entreprendre. Cette observation justifie notre modélisation théorique qui consiste à étudier des relations contractuelles contraintes à la fois par des problèmes de sélection adverse mais aussi par des problèmes de risque moral.

Un premier objectif de notre travail est alors d'expliquer le choix des contrats par les autorités organisatrices de transport ainsi contraintes d'un point de vue informationnel. La Nouvelle Théorie de la Régulation (Baron and Myerson [1982] et Laffont and Tirole [1986]) propose un cadre normatif permettant d'appréhender les relations contractuelles entre entreprises réglementées et autorités publiques au travers du prisme offert par la Théorie des Incitations. Dans ces modèles, les autorités offrent des régulations incitatives leur permettant de réduire ainsi les asymétries d'information inhérentes au contrat et d'inciter les opérateurs à réduire efficacement leurs coûts. Un message fondamental de cette littérature est que les régulations incitatives optimales résultent d'un arbitrage entre d'une part la recherche de l'efficacité allocative et d'autre part le désir de l'autorité publique de réduire les rentes informationnelles des opérateurs. Intuitivement, les mécanismes de type «coûts du service» limitent les incitations des entreprises à réduire ces coûts et limitent d'autant leurs rentes informationnelles. A contrario, et bien qu'ils abandonnent souvent des rentes excessives aux opérateurs, les mécanismes de type «prix fixe» ont de fortes vertus incitatives puisqu'ils permettent à l'opérateur de bénéficier de tous les efforts qu'il peut exercer pour réduire ses coûts. En supposant que les autorités publiques ont une forte capacité d'engagement, cet arbitrage est théoriquement résolu par le choix d'une régulation optimale de second rang, qui peut être (sous certaines conditions techniques) mis en oeuvre grâce à un menu complexe de contrats linéaires. ${ }^{6}$

La littérature empirique a, quant à elle, supposé jusqu'à présent que les contrats observés en pratique sont tous issus de mécanismes optimaux de ce type (Wolak [1994], Wunsch [1994], et Gasmi, Laffont and Sharkey [1997] par exemple). Plus récemment, le

\footnotetext{
${ }^{5}$ Rogerson [2003] observe qu'un tel choix dichotomique est tout à fait susceptible d'approcher l'optimum que l'on pourrait obtenir avec des menus de contrats plus complexes.

${ }^{6}$ Voir Laffont et Tirole [1993].
} 
débat s'est porté sur la pertinence de telles hypothèses et sur les performances de menus de contrats plus parcimonieux. En effet, la mise en oeuvre de menus complexes de contrats linéaires n'est pas toujours possible dès lors que les autorités publiques ne disposent pas des capacités techniques ou le degré d'expertise requis.

Dans l'industrie du transport urbain, nous n'observons en pratique que deux types de contrats aux propriétés significativement différentes en termes d'incitations à la réduction des coûts d'exploitation. Il est donc difficilement envisageable de supposer que ces contrats sont issus de menus relativement complexes de contrats optimaux. Nous proposons donc dans un premier temps de construire un mécanisme de réglementation optimal qui s'adapte au contexte du transport urbain français. Ici, l'autorité propose à l'opérateur un menu simple de contrats qui peuvent être soit de type «coût du service» soit de type «prix fixe». L'argument justifiant ce choix restreint est développé par Wilson [1993] et Rogerson [2003]: Un menu simple de deux contrats peut déjà permettre à une autorité organisatrice d'obtenir une part importante du bien-être social généré par des menus plus complexes. Dans un tel cadre, les opérateurs les plus efficaces sont conduits à adopter des mécanismes à «prix fixes» et bénéficient de fait de rentes informationnelles importantes. Les opérateurs les moins efficaces s'orientent vers des contrats de type «coût du service».

Une différence importante avec l'analyse de Rogerson [2003] est que notre modèle théorique est dynamique et s'attache à caractériser les évolutions dans les contrats passés entre un même opérateur et une autorité publique donnée. L'industrie du transport urbain en France est en effet caractérisée par une augmentation continue des subventions versées par les autorités aux opérateurs, indépendamment des caractéristiques du service, de l'identité de l'autorité ou de l'opérateur. Nous justifions cette tendance par le fait que les autorités peuvent souffrir d'une capacité limitée d'engagement à la nonrenégociation des termes du contrat. Cette capacité d'engagement limitée est à la source des incitations des autorités publiques à augmenter les subventions au cours du temps, à mesure que les capacités productives de l'opérateur sont mieux appréhendées au vu de ses performances passées. Nous suggérons également que la mise en place en 1993 de procédures d'appel d'offre pour l'adjudication des opérations de transport n'a pas eu l'effet attendu sur le renouvellement des opérateurs et l'augmentation de la concurrence ex ante. Une conséquence immédiate est qu'une même entreprise est chargée des opérations de transport d'une période à l'autre. Les relations entre les autorités organisatrices et les opérateurs sont donc similaires à des relations de long terme entrecoupées de périodes de 
«re-négociation». Nous reviendrons en détail sur ces aspects.

L'argumentaire théorique sous-tendant cette explication est bien connu de la littérature sur l'engagement contractuel limité ${ }^{7}$ mais il est sans doute bon d'en rappeler les principales articulations. Dès lors que l'autorité publique souffre d'une connaissance imparfaite des caractéristiques des opérateurs, offrir un menu simple de contrats permet une certaine auto-sélection des opérateurs suivant leur efficacité. Comme nous l'avons vu ci-dessus, les opérateurs les plus efficaces sélectionnent les contrats à «prix fixes» alors que les moins efficaces préfèrent les contrats «coûts du service». L'adoption par un opérateur donné d'un tel contrat est donc interprétée par l'autorité publique comme étant une information plutôt défavorable. Dans un cadre dynamique où les relations contractuelles entre opérateurs et autorités publiques couvrent plusieurs périodes, il est tout à fait naturel que ces dernières prennent en compte l'information révélée par le passé par les opérateurs dans leurs choix de contrats pour réajuster les termes du nouveau contrat. Accroître les subventions associées aux contrats à «prix fixes» permet à l'autorité de s'ajuster et à des entreprises a priori moins efficaces d'introduire elles aussi certains gains de productivité. Ces gains doivent toutefois être évalués d'un point de vue dynamique : Les opérateurs les plus efficaces peuvent préférer ainsi adopter des contrats de type «coûts du service» dans les premières périodes de la relation afin de bénéficier de l'accroissement des subventions que la renégociation de ces contrats initiaux induira. Dans un monde où les autorités publiques ont une capacité d'engagement limitée, l'arbitrage entre efficacité allocative et rente informationnelle doit être reconsidéré d'un point de vue dynamique : L'information sur les opérateurs n'est révélée que graduellement et, simultanément, les subventions aux opérateurs croissent au cours du temps.

Notre second objectif est de proposer une validation empirique de notre modèle théorique à l'aide d'une base de données riche en observations sur les caractéristiques des réseaux de transports, les autorités organisatrices, et les opérateurs du service (49 réseaux de transport urbain sur la période 1987-2001). Nous construisons un modèle micro-économétrique du choix du type de contrat par les autorités organisatrices et de la réponse optimale des opérateurs. En particulier, nous nous concentrons sur le caractère dynamique des arrangements contractuels proposés par les autorités et sélectionnés par les opérateurs. Nous montrons comment le choix des dynamiques contractuelles dépend des caractéristiques intrinsèques de l'opérateur. Ainsi, nous suggérons que les opérateurs de transport

\footnotetext{
${ }^{7}$ Voir par exemple Laffont et Martimort (2002, Chapitre 9).
} 
urbain participent activement au choix des schémas réglementaires. La méthodologie employée est similaire à celle utilisée par les économistes qui étudient les choix d'entrée des entreprises dans des marchés de type concurrence oligopolistique (Hotz et Miller [1993], Bajari et alii [2007], et Ryan [2007]).

Nous évaluons la distribution de l'inefficacité des opérateurs de transport, et suggérons qu'une bonne modélisation de la capacité d'engagement des autorités organisatrices est fondamentale pour une évaluation satisfaisante des capacités productives des opérateurs et donc de l'efficacité de la gestion déléguée. En d'autres termes, les paramètres d'efficacité du secteur ne peuvent être évalués précisément qu'en prenant en compte non seulement les contraintes informationnelles mais aussi les contraintes institutionnelles qui constituent le «paysage du contrat».

L'article est organisé de la manière suivante. Dans la Section 2, nous présentons plus en détails l'industrie du transport urbain en France. La Section 3 est consacrée au développement de notre modèle théorique et motive nos choix de modélisation. La Section 4 présente les données disponibles et l'application empirique.

\section{L'industrie du transport urbain en France}

Le transport urbain est un service public dont la politique est déterminée par une entité publique, l'autorité organisatrice, tandis que l'exploitation peut être confiée à une entreprise privée, l'exploitant ou opérateur. Les relations entre les deux parties sont régies par un contrat qui définit le type de réglementation adopté.

La mise en oeuvre de la Loi d'Orientation des Transports Intérieurs (loti) en 1982 résulte d'une volonté générale de réglementation du droit des transports. Divers principes en sont issus, notamment l'organisation de la politique de transport par les collectivités locales, la définition du périmètre de transport, le financement par les bénéficiaires directs et autres bénéficiaires privés ou publics indirects etc. Une conséquence importante de cette loi est que les autorités locales sont responsables du financement du transport urbain à partir de cette date.

Les deux parties sont liées par un contrat qui précise la nature des services à fournir par l'exploitant, les conditions de l'exploitation du service, le type de financement et les modalités de contrôle de l'utilisation des fonds engagés. En particulier, l'autorité détermine dans le contrat la structure du réseau de transport, la capacité et la qualité du service, la tarification, les niveaux de subvention, et le niveau de l'investissement. Le 
prix du transport imposé est bien inférieur au prix qui permettrait d'assurer l'équilibre financier de l'exploitation qui est déficitaire en permanence. Il est donc nécessaire de subventionner l'activité pour assurer son équilibre financier.

En principe, depuis l'introduction de la loi Sapin en 1993, l'autorité doit mettre en place un système d'appel d'offre pour choisir l'opérateur de transport de sa zone urbaine. En pratique cependant, très peu de réseaux changent d'opérateurs, comme indiqué ci-dessous. Plusieurs raisons peuvent expliquer ce phénomène : Les autorités de transport ne disposent pas d'un niveau d'expertise suffisant pour la mise en place de systèmes d'appel d'offre efficaces, ou elles ne souhaitent pas réaliser d'effort important pour garantir le succès de ces systèmes. D'autre part, les opérateurs appartiennent tous à des groupes industriels importants qui fournissent généralement d'autres services municipaux comme la distribution de l'eau ou le ramassage des ordures. Une telle implication peut compliquer la tâche du régulateur désireux de se défaire de son opérateur en cas de mauvaise performance.

L'autorité et l'opérateur sont donc conscients qu'ils sont amenés à collaborer sur plusieurs périodes contractuelles. Lorsqu'un contrat se termine, il est probable que l'autorité considère que l'appel d'offre est une opportunité pour «re-négocier»le contrat. Cette relation de long terme motive la structure dynamique de notre modèle comme présenté ci-dessous.

Deux grands types de contrats, qui diffèrent par l'allocation des recettes commerciales et la nature du remboursement des coûts d'exploitation, sont utilisés en pratique. Le premier type, intitulé «coût du service», définit une convention où l'exploitant, à l'issue de l'exercice, cède les recettes commerciales à l'autorité, qui lui rembourse en contrepartie la totalité de ses coûts ex-post. L'exploitant n'a donc aucune incitation à fournir un effort pour réduire ses coûts de production. En revanche, dans le cas des contrats de type «prix fixe», l'exploitant conserve les recettes commerciales et obtient un remboursement forfaitaire de ses coûts qui est fixé ex-ante. ${ }^{8}$ L'opérateur est responsable des dépassements potentiels des coûts réels au dessus du niveau des coûts espérés. Il a donc intérêt à fournir un niveau d'effort optimal pour la réduction des coûts d'exploitation.

En général, les contrats sont signés pour une période de 5 ans en moyenne, ce qui nous permet d'observer des séries de plusieurs contrats pour chaque réseau. Nous observons un

\footnotetext{
${ }^{8}$ Nous rassemblons sous le même nom «prix fixe» les contrats de type «compensation forfaitaire», et ceux de type «prix forfaitaire». Bien que ces deux régimes réglementaires impliquent des mécanismes de garantie des recettes commerciales différents, ils ont les mêmes effets en termes d'incitations à la réduction des coûts. Ce sont ces effets qui nous intéressent ici.
} 
total de 136 contrats. Pour un même réseau, le schéma réglementaire peut changer d'une période à l'autre. Ainsi, nous observons 17 passages de contrats type coût du service à des contrats de type prix fixe, contre 3 passages de prix fixe à coût du service. Enfin, notons que seuls 2 réseaux changent d'opérateur au cours de la période, ce qui nous permet de supposer que la mise en place d'appels d'offre a eu très peu d'effet sur la mobilité des opérateurs d'un réseau à l'autre. En revanche, nous détectons 22 changements de régulateur au cours de la même période.

Une particularité intéressante de l'industrie est que nous observons une croissance continue des subventions par unité d'offre entre 1987 et 2001 dans plus de la moitié des réseaux. Notons que cette croissance est indépendante des caractéristiques du réseau, des autorités, des opérateurs, ou de l'inflation. Le Graphique 1 présente une mesure de la subvention par unité d'offre (par siège-kilomètre) corrigée de la hausse des prix des facteurs de production pour un échantillon de dix réseaux. ${ }^{9}$

La croissance de la subvention suggère que les termes des contrats de transport ne sont pas constants d'une période à l'autre. Il est donc vraisemblable que les autorités ne sont pas capables de s'engager à ne pas utiliser l'information acquise d'une période à l'autre pour renégocier ces contrats. Nous verrons au cours de cet article comment des hypothèses alternatives quant à la capacité de l'autorité à s'engager ou non peuvent affecter les résultats empiriques. Ainsi, nous suggérons qu'une bonne définition du comportement des autorités publiques est fondamentale pour bien comprendre le fonctionnement de cette industrie.

\section{Le modèle théorique}

Le modèle théorique que nous développons s'appuie sur les leçons de la Nouvelle Théorie de la Régulation mais l'adapte aux caractéristiques du secteur et en particulier au choix binaire entre deux types de contrats. Les contrats de type prix fixe se résument à une subvention fixe $b$. Les contrats de type coûts du service correspondent à une subvention que nous normaliserons à zéro et à un remboursement total des coûts pour l'opérateur.

\footnotetext{
${ }^{9} \mathrm{La}$ croissance des subventions peut-être due à l'expansion des réseaux vers des zones urbaines moins denses où la demande est plus faible et plus incertaine. De tels effets sont sans doute ponctuels et il est peu probable que l'incertitude sur la demande subsiste dans le long terme. Notons par exemple que le taux de croissance sur 15 ans des revenus commerciaux par unité d'offre pour ces dix réseaux est positif dans une large majorité de cas : Angers : $+4.9 \%$, Annecy : $+33 \%$, Belfort : $+12.9 \%$, Bourges : $-37 \%$, Caen : $+38.6 \%$, Le Havre : $+2 \%$, Limoges : $-6.6 \%$, Lorient : $-21.1 \%$, Toulouse : $+68.5 \%$, et Lens : $+25.5 \%$.
} 
L'autorité publique a des préférences définies comme suit:

$$
W=S-(1+\lambda) t+\alpha U \quad \text { où } 0 \leq \alpha<1+\lambda \quad \text { et } \lambda>0 .
$$

$S$ correspond à la valeur sociale du transport que nous supposerons indépendante du prix du service dans un cadre où l'élasticité de la demande est très faible. La subvention $t \in\{c, b\}$ dépend comme nous l'avons signalé du type de contrat signé. $\lambda>0$ est un paramètre qui représente le coût des fonds publics. Enfin, et suivant en cela Baron et Myerson [1982], l'autorité publique accorde un poids $\alpha$ au profit de l'entreprise dans son objectif. Ce poids peut représenter l'influence politique de l'entreprise au niveau local, refléter les préférences de la majorité politique en place plus ou moins prête à favoriser des arbitrages en faveur de l'opérateur ${ }^{10} \mathrm{ou}$, enfin, être un index du pouvoir de marchandage de l'entrepreneur dans l'attribution des contrats. ${ }^{11}$ Pour rester dans une constellation de paramètres pour laquelle les rentes sont coûteuses nous supposerons donc que $\alpha<1+\lambda$. Cette condition implique aussi que les contrats de gestion déléguée doivent arbitrer entre l'abandon aux opérateurs de rentes informationnelles coûteuses et recherche de l'efficacité allocative au travers d'incitations conduisant à la réduction des coûts du service.

Le coût observable du service $C$ inclut à la fois des dimensions de sélection adverse et de risque moral qui sont toutes deux non-observables. $\theta$ représente un paramètre d'efficacité a priori de l'entreprise et $e$ désigne son effort managérial:

$$
C=\theta-e .
$$

L'effort managérial est coûteux pour l'opérateur et la désutilité correspondante sera dénotée par $\psi(e)\left(\psi^{\prime}>0, \psi^{\prime \prime}>0\right)$ avec $\psi(0)=0$.

Le paramètre d'efficacité $\theta$ est tiré dans l'intervalle $[\underline{\theta}, \bar{\theta}]$ selon la loi $F(\cdot)$ de densité $f(\cdot)$. Nous supposerons la monotonie du taux de hasard, i.e., $\frac{d}{d \theta}(R(\theta))>0$ où $R(\theta)=\frac{F(\theta)}{f(\theta)}$. $^{12}$

\footnotetext{
${ }^{10}$ Dans ce cas, le paramètre $\alpha$ pourra refléter la capacité de l'opérateur à corrompre le décideur public.

${ }^{11} \mathrm{~A}$ ce titre, il est important de noter que ce secteur est sujet à très peu de concurrence ex ante. Comme il a été noté dans la Décision $n^{0}$ 05-D-38 du Conseil de la Concurrence, le risque de collusion entre opérateurs potentiels est important et est reflété par le peu de concurrents prêts à enchérir pour un contrat donné. Dans plus de $60 \%$ des cas, il n'y a qu'un enchérisseur. Nous remercions un rapporteur pour avoir porté à notre attention cette caractéristique du secteur. Notre modélisation capture ces aspects de corruption verticale ou de peu de concurrence horizontale de manière ad hoc au travers du paramètre $\alpha$. Cette ad hocité nous permet alors de développer des aspects dynamiques du modèle qui deviendraient intractable si nous avions porté une attention plus soutenue aux aspects collusifs.

${ }^{12} \mathrm{Il}$ est important de noter qu'une même entreprise opérant sur des municipalités différentes pourra a priori avoir des paramètres d'efficacité distincts sur chacun de ces contrats. Cette hypothèse capture le fait que les conditions d'efficacité du service sont, en première approximation, dans une assez large mesure idiosyncratiques au secteur desservi (puisqu'elles dépendent étroitement de la structure du réseau,
} 
Contrats dynamiques avec engagement : Supposons dans un premier temps que l'autorité publique puisse s'engager à offrir un menu de contrats de long-terme couvrant deux périodes. Cette hypothèse nous permet d'obtenir une borne supérieure des gains contractuels accessibles aux deux parties, ce qui se traduira dans notre analyse empirique en termes d'un biais sur les gains de l'échange.

Un tel menu de contrats avec engagement consiste donc en un profil de «prix fixes» pour chacune des périodes, et si à l'une de ces périodes un tel contrat à «prix fixe» n'est pas choisi par l'opérateur, ce dernier optera pour le contrat «coûts du service» sur la période correspondante.

Désignons par $\delta$ le facteur d'escompte et normalisons le poids comptable de la première période dans le profil intertemporel des gains en introduisant le paramètre $\beta=\frac{1}{1+\delta}$.

Un contrat à prix fixe de long terme correspond à des subventions $b_{1}$ and $b_{2}$ dans chacune des périodes. Avec de tels contrats, l'entreprise choisit l'effort de premier rang $e^{*}$ tel que $\psi^{\prime}\left(e^{*}\right)=1$. Désignant la valeur sociale nette de cet effort par $k=e^{*}-\psi\left(e^{*}\right)$, un tel contrat à «prix fixes» de long terme est choisi par l'entreprise quand ses coûts sont suffisamment faibles, i.e.,

$$
\theta \leq \beta b_{1}+(1-\beta) b_{2}+k=\theta^{*}
$$

La maximisation du bien-être espéré conduit à la détermination d'un «cut-off» optimal dans l'intervalle des paramètres d'efficacité:

Proposition 1 (Gagnepain, Ivaldi, Martimort [2008]): Avec engagement total, le contrat $\grave{a}$ prix-fixe optimal correspond à des subventions constantes au cours du temps, $b_{1}^{F}=b_{2}^{F}=$ $b^{F}: 13$

$$
k=\left(1-\frac{\alpha}{1+\lambda}\right) R\left(b^{F}+k\right) .
$$

Seuls les firmes les plus efficaces choisissent ce contrat

$$
\theta \leq \theta^{F}=b^{F}+k
$$

Les plus inefficaces optent quant à elles pour un contrat de coûts du service.

Un contrat à «prix fixe» avec une subvention suffisamment importante assurerait que l'opérateur, quel que soit son efficacité, exerce toujours un effort de premier rang et des conditions de trafic, de la topographie de la ville, etc). De la même manière, nous supposerons aussi en première approximation que tous les opérateurs, quel que soit l'identité de l'entreprise en question verront leurs coûts être tirés de la même loi $F(\cdot)$.

${ }^{13}$ Le résultat que la dynamique des subventions est stationnaire est classique. Voir Laffont and Martimort (2002, Chapitre 8) par exemple. 
obtienne les gains de productivité associés. Ce serait bien sûr très coûteux du point de vue des rentes informationnelles ainsi abandonnées au secteur privé. Un contrat de type «coût du service» conduit à diminuer ces rentes mais c'est au prix de l'absence de toute incitation à réduire les coûts. Le choix de la subvention optimale $b^{F}$ traduit l'arbitrage entre extraction des rentes informationnelles de l'opérateur et recherche de l'efficacité.

Il est intéressant de noter qu'un poids plus important sur les profits de l'opérateur ( $\alpha$ plus grand) dans la fonction objectif de l'autorité publique (que ce soit du fait d'une «influence» politique plus affirmée ou d'une concurrence diminuée) conduit à favoriser les rentes de l'opérateur dans cet arbitrage, ce qui se traduit de facto par un accroissement des subventions. D'autre part, des gains de productivité associés à une valeur sociale supérieure ( $k$ plus grand) conduisent aussi à accroître les subventions.

Contrats dynamiques avec renégociation : L'hypothèse d'engagement qui a été la nôtre jusqu'à présent est vraisemblablement excessive. En pratique, le peu de concurrence entre opérateurs potentiels à chaque renouvellement des délégations comme le rythme des échéances électorales auxquelles sont soumis les engagements contractuels, conduit plutôt à considérer la relation contractuelle entre autorité publique et un même opérateur comme représentée par des contrats qui, s'ils apparaissent comme de long terme, n'en demeurent pas moins renégociés ou «négociés à nouveau» entre ces mêmes partenaires lorsqu'arrivés à échéance.

D'un point de vue théorique, la renégociation des contrats est définie comme résultant du commun bon vouloir des parties contractantes de changer les termes de leur accord dès lors qu'elles y trouvent un avantage mutuel au gré des informations qui ont pu être révélées au cours du temps. ${ }^{14}$ Cette définition couvre donc la modification des contrats de long-terme à des dates ex interim mais aussi, lorsque pris dans une acceptation plus large, la possibilité pour des contractants dont les pouvoirs de marchandage à une date donnée sont liés implicitement aux contrats passés de re-négocier de futurs accords et d'anticiper ces négociations futures dans leurs accords initiaux. C'est certainement cette dernière interprétation qui correspond le mieux au cadre institutionnel qui nous intéresse ici.

Dès lors que l'autorité publique souffre d'une connaissance imparfaite des caractéristiques des opérateurs, offrir un menu simple de contrats permet une certaine auto-sélection des opérateurs suivant leur efficacité. Comme nous l'avons vu ci-dessus dans le cas

\footnotetext{
${ }^{14}$ Voir par exemple Laffont et Martimort (2002, Chapitre 9) et les articles qui y sont référencés.
} 
d'engagement, les opérateurs les plus efficaces sélectionnent les contrats à «prix fixes» alors que les moins efficaces préfèrent les contrats «coûts du service». L'adoption par un opérateur donné d'un tel contrat est donc interprétée par l'autorité publique comme étant une information plutôt défavorable. Dans un cadre dynamique où les relations contractuelles entre opérateurs et autorités publiques couvrent plusieurs périodes, il est tout à fait naturel que ces dernières prennent en compte l'information révélée par le passé par les opérateurs dans leur choix de contrats pour réajuster les termes du nouveau contrat. Accroître les subventions associées aux contrats à «prix fixes» permet à l'autorité de s'ajuster à des entreprises a priori moins efficaces et d'introduire ainsi de nouveaux gains de productivité. Ces gains doivent toutefois être évalués d'un point de vue dynamique: Les opérateurs les plus efficaces peuvent préférer ainsi adopter des contrats de type «coûts du service» dans les premières périodes de la relation afin de bénéficier de l'accroissement des subventions que la renégociation de ces contrats initiaux induira.

Une fois qu'un contrat de long terme à «prix fixes» $\left\{b_{1}, b_{2}\right\}$ a été offert et, éventuellement refusé par une firme pas assez efficace, l'autorité publique a des incitations à offrir pour la deuxième période un contrat renégocié. Cette renégociation, pour être attractive auprès de l'opérateur, se doit donc d'accroître les subventions. Anticipant cette issue, les firmes de coûts intermédiaires peuvent refuser les contrats de «prix fixes» initiaux préférant alors les contrats de type coûts du service, attirées ainsi par les rentes futures qu'elles peuvent obtenir dans des contrats à «prix fixes» plus généreux dans le futur.

La résolution de l'équilibre de ce jeu de contrats dynamiques est complexe et nous renvoyons à Gagnepain, Ivaldi, Martimort [2008] pour une caractérisation détaillée. Néanmoins, et pour faciliter ici la compréhension, il est utile de préciser les stratégies des acteurs:

- Autorité publique: Elle s'engage à un menu de contrats de long terme correspondant à des subventions $\left\{b_{1}, b_{2}\right\}$ dans le cas des «prix fixes», ${ }^{15}$ et une offre renégociée $\tilde{b}_{2}$ qui peut remplacer $b_{2}$ à la date 2 . Cette renégociation est conditionnelle aux croyances révisées sur le type de l'entreprise, révision qui prend en compte l'observation de sa décision d'accepter ou non la subvention $b_{1}$ dès la première période.

- Opérateur: L'opérateur adopte une stratégie de «cut-off». Il accepte l'offre $\left\{b_{1}, b_{2}\right\}$

\footnotetext{
${ }^{15}$ Notons que l'engagement sur $b_{2}$ peut être implicite si l'on adopte comme nous le suggérons ci-dessus le point de vue que l'autorité publique offre des contrats limités dans le temps. $b_{2}$ apparaît alors comme une promesse pour le futur. Notre point de vue est que la relation répétée entre contractants rend de telles promesses même si elles sont implicites et «cachées» tout à fait crédibles.
} 
si et seulement si $\theta \in \Theta_{1}=\left[\underline{\theta}, \theta_{1}^{*}\right]$. Les firmes ayant un type $\theta \in \Theta_{2}=\left[\theta_{1}^{*}, \theta_{2}^{*}\right]$ $\left(\theta_{2}^{*} \geq \theta_{1}^{*}\right)$ acceptent seulement l'offre renégociée $\tilde{b}_{2}$. Celles de types $\theta \geq \theta_{2}^{*}$ choisissent toujours le contrat de remboursement des coûts dans chacune des périodes.

Un profil de subventions $\left\{b_{1}, b_{2}\right\}$ est robuste à la possibilité d'une renégociation si et seulement si l'autorité publique trouve optimal de ne pas perturber les termes du contrat une fois acquise l'information sur les coûts de l'entreprise révélée même partiellement par les choix de première période. Un contrat est donc robuste dès lors que la promesse $b_{2}$ est réellement le contrat mis en oeuvre à la date 2. Ce sont ces profils de subventions qui attireront notre attention. ${ }^{16}$

Pour que les stratégies ci-dessus constituent un équilibre, il faut que le type $\theta_{1}^{*}$ soit indifférent entre choisir dès la date 1 le profil de subventions $\left\{b_{1}, b_{2}\right\}$ correspondant à des contrats à «prix fixes» dans chacune des périodes et choisisse d'abord un contrat «coûts du service» avant d'opter seulement à la date 2 pour le contrat à «prix fixe» correspondant à la subvention $b_{2}$ :

$$
\beta b_{1}+(1-\beta) b_{2}+k-\theta_{1}^{*}=(1-\beta)\left(b_{2}+k-\theta_{1}^{*}\right) \text { ou } \theta_{1}^{*}=b_{1}+k
$$

De la même manière, on trouve que nécessairement:

$$
\theta_{2}^{*}=b_{2}+k
$$

Les contrats robustes à la renégociation peuvent donc être caractérisés comme suit:

Proposition 2 (Gagnepain, Ivaldi, Martimort [2008]): Un profil de subventions $\left(b_{1}, b_{2}\right)$ est robuste à la renégociation si et seulement si:

$$
b_{2} \geq b^{F} \text { et } k f\left(b_{2}+k\right)-\left(1-\frac{\alpha}{1+\lambda}\right)\left(F\left(b_{2}+k\right)-F\left(b_{1}+k\right)\right) \leq 0 .
$$

La première de ces conditions indique que l'autorité publique n'est pas désireuse de changer les termes du contrat, une fois observé que la firme a accepté un contrat à prix fixe dès la première période. Ce choix est en effet interprété comme étant une «bonne nouvelle» sur les coûts de l'opérateur. Accroître les subventions ne sert alors à rien.

\footnotetext{
${ }^{16}$ En effet, il est assez facile de constater qu'un Principe de Robustesse à la Renégociation existe dans notre contexte. Supposons que l'autorité veuille effectivement renégocier à la date 2 vers une offre différente de ses promesses initiales (formellement, $\left.\tilde{b}_{2} \neq b_{2}\right)$. Considérons le nouveau contrat $\left(b_{1}, \tilde{b}_{2}\right)$. Ce profil de subventions est maintenant crédible car s'il existait une autre subvention $\bar{b}_{2} \neq \tilde{b}_{2}$ préférée à la date 2 à $\tilde{b}_{2}$ conditionnellement aux croyances à cette date, nous obtiendrions une contradiction avec l'optimalité supposée de $\tilde{b}_{2}$.
} 
La deuxième de ces conditions indique, quant à elle, que l'autorité publique n'est pas désireuse de modifier ses promesses sur les subventions de second période dès lors que le profil de subventions correspondant à des contrats à «prix fixes» a été refusé dès la première période. De manière assez intuitive, cette condition confirme que l'autorité publique n'est pas désireuse d'augmenter d'un montant $d b$ les subventions de seconde période et d'obtenir des gains de productivité (en quantité espérée $(1+\lambda) k f\left(b_{2}+k\right) d b$ ) dès lors que l'accroissement du coût social des rentes informationnelles de toutes les firmes prenant ce contrat à «prix fixe» en second période est suffisamment important (terme $\left.(1+\lambda-\alpha)\left(F\left(b_{2}+k\right)-F\left(b_{1}+k\right)\right)\right)$.

Il est important de noter que cette condition de robustesse à la renégociation implique que nécessairement les profils de subvention sont croissants et qu'en corollaire la révélation d'information sur l'efficacité des opérateurs ne peut être que graduelle.

Désignons par $\mu$ le multiplicateur de la contrainte de renégociation (5) dans l'optimisation du bien-être espéré intertemporel, nous obtenons:

Proposition 3 (Gagnepain, Ivaldi, Martimort [2008]): Le contrat optimal robuste à la renégociation $\left\{b_{1}^{R}, b_{2}^{R}\right\}$ correspond à un sentier de subventions croissantes:

$$
b_{1}^{R}<b^{F} \text { avec } b_{1}^{R}<b_{2}^{R}
$$

et

$$
\begin{gathered}
k-\frac{\mu}{\beta(1+\lambda)}\left(1-\frac{\alpha}{1+\lambda}\right)=\left(1-\frac{\alpha}{1+\lambda}\right) R\left(b_{1}^{R}+k\right) ; \\
k+\frac{\mu}{(1-\beta)(1+\lambda)}\left(1-\frac{\alpha}{1+\lambda}\right)-\frac{\mu k}{(1-\beta)(1+\lambda)} \frac{f^{\prime}\left(b_{2}^{R}+k\right)}{f\left(b_{2}^{R}+k\right)}=\left(1-\frac{\alpha}{1+\lambda}\right) R\left(b_{2}^{R}+k\right) ;
\end{gathered}
$$

et

$$
-k f\left(b_{2}^{R}+k\right)+\left(1-\frac{\alpha}{1+\lambda}\right)\left(F\left(b_{2}^{R}+k\right)-F\left(b_{1}^{R}+k\right)\right)=0 .
$$

\section{Le modèle empirique}

Nous présentons maintenant la partie empirique de notre étude. Nous proposons ici une estimation de la distribution du paramètre d'efficacité $\theta$ inobservable des opérateurs de transport urbain en France. Nous montrons comment cette distribution dépend des caractéristiques intrinsèques des entreprises. Nous suggérons également que cette inefficacité est sous-évaluée s'il est supposé à tort que les termes du contrat de transport sont inchangés d'une période à l'autre (l'autorité s'engage à ne rien modifier), en comparaison 
avec la situation actuelle où l'autorité augmente les subventions comme prédit par notre modèle théorique.

Notre hypothèse principale consiste à dire que les autorités utilisent actuellement en France des contrats robustes à la renégociation. Ceci implique que nous devons observer dans notre base de données les séries de contrats suivantes:

- Une série $(P P)$ de contrats de type «prix fixe». Il s'agit d'une situation où l'autorité publique contracte avec un opérateur efficace $\left(\theta \leq \theta_{1}^{*}\right)$ suivant un profil de subventions robuste à la renégociation.

- Une série $(C P)$ de contrat de type «coût du service» suivi d'un contrat de type prix fixe. Dans ce cas, l'autorité publique supervise un opérateur à l'efficacité moyenne $\left(\theta_{1}^{*} \leq \theta \leq \theta_{2}^{*}\right)$.

- Une série $(C C)$ de contrats de type «coût du service»: Ici, l'opérateur réglementé est plutôt inefficace $\left(\theta \geq \theta_{2}^{*}\right)$.

Nous comparons cette situation réelle à un scénario hypothétique où l'autorité ne modifie pas et s'engage sur les termes du contrat d'une période à l'autre. Un tel scénario impliquerait observer les séries de contrats suivantes: ${ }^{17}$

- Une série $(P)$ de contrats de type «prix fixe». Ce type de réglementation serait appliquée à des opérateurs efficaces $\left(\theta \leq \theta_{F}\right)$.

- Une série $(C)$ de contrats de type «coût du service» avec un opérateur plutôt inefficace $\left(\theta \geq \theta_{F}\right)$.

Une fois définies les différentes séries de contrats, nous pouvons présenter les données disponibles et leur organisation, propre à chaque arrangement contractuel, au moment de l'estimation du choix de contrat et de la distribution de l'inefficacité des opérateurs.

\footnotetext{
${ }^{17}$ Notons que nous observons en réalité dans notre base de données une majorité de réseaux où les subventions croissent d'une période à l'autre. Nous observons également une minorité de réseaux où les subventions sont constantes.

Identifier les deux types d'engagement à partir des contrats prix fixes est facile, il suffit d'observer l'évolution des subventions. Malheureusement, l'observation d'un contrat de type coût du service n'apporte aucune information à ce niveau (puisque les subventions s'alignent sur les coûts réels et non sur des objectifs fixés ex ante par l'autorité). Nous sommes donc contraints par un problème d'identification qui nous oblige à considérer que tous les réseaux sont caractérisés par un engagement limité de l'autorité. Notre contribution est significative dans le sens où la littérature empirique sur la régulation des monopoles considère généralement que les autorités font preuve d'engagement. Nous montrons comment le passage d'une hypothèse à l'autre modifie les résultats empiriques.
} 


\subsection{Les données}

Nous disposons d'observations sur 49 opérateurs de réseaux urbains pour la période 19872002. Une observation est la réalisation d'un contrat de transport dans un réseau spécifique au cours d'une année. Ces données sont éditées annuellement par le Centre d'Etude et de Recherche du Transport Urbain (certu), et le Groupement des Autorités Responsables du Transport (gart). Pour notre étude, nous avons sélectionné uniquement les zones urbaines avec plus de 100000 habitants afin de réduire les risques d’hétérogénéité entre les différents réseaux.

Le Tableau 1 présente chacune des données disponibles. Les coûts totaux indiquent le volume total des coûts qui n'est pas couvert par les revenus commerciaux. ${ }^{18}$ Les subventions incluent les sommes versées en début d'exercice à l'opérateur pour équilibrer le budget prévisionnel, plus les sommes versées à l'opérateur en fin d'activité pour rembourser intégralement ses coûts de production. En divisant le total des subventions par la quantité de places-kilomètres offertes par l'opérateur, nous obtenons une mesure de la subvention versée pour chaque unité de production offerte. La taille du réseau est la somme des longueurs de toutes les lignes du réseau. Enfin, nous disposons pour chaque entreprise de la part des ingénieurs sur le nombre total d'employés.

A ce premier groupe de variables, nous ajoutons plusieurs variables muettes qui nous permettent de prendre en compte les caractéristiques des opérateurs: Ainsi, nous sommes capables d'identifier le groupe de services municipaux auquel appartient chaque opérateur, à savoir, Transdev, Connex, Keolis, et Agir.

Définition d'une période contractuelle et sélection des données: Notre base de données contient 49 réseaux observés entre 1987 et 2001, ce qui correspond à 136 contrats. Comme la durée d'une période contractuelle est de 5 années en moyenne, on observe généralement des séries de trois contrats par réseau entre 1987 et 2001. Très peu de réseaux présentent des séries de 1,2 , ou 4 contrats.

La sélection de l'échantillon pertinent pour l'estimation dépend étroitement du type de scénario contractuel envisagé: ${ }^{19}$

\footnotetext{
${ }^{18}$ Notons que notre modèle théorique utilise une simplification comptable en considérant que les revenus commerciaux sont récupérés par l'autorité organisatrice et que les coûts d'exploitation sont remboursés à l'opérateur. Dans nos données, les subventions observées sont les différences entre les coûts et les revenus. Afin de faire coïncider nos données avec notre modèle nous considérons les coûts d'exploitation nets des revenus commerciaux.

${ }^{19}$ Notons qu'un contrat dans un réseau devrait en principe correspondre à une unique observation dans notre modèle théorique, i.e., les objectifs du contrat devraient rester constants au cours des - par exemple - 5 ans de la période contractuelle. La réalité des données peut être quelque peu différente. En pratique,
} 
- Engagement total: Ici, les arrangements contractuels impliquent des séries de contrats qui seraient en principe identiques. Dans notre modèle de choix de contrat (Tableau 2), nous modélisons simplement la probabilité de choix d'un contrat prix fixe. Ainsi, notre variable expliquée dans ce cas est égale à 1 si le contrat observé au cours d'une unique période contractuelle est de type prix fixe. Nous considérons dans ce cas tous les contrats de notre base de données.

- Robustesse à la renégociation: Nous nous concentrons ici sur des séries de deux contrats. Les trois possibilités de séries sont $(F F)$, $(C F)$, et $(C C)$. Ainsi nous expliquons le choix d'une série de contrats plutôt qu'une autre et montrons comment cette préférence dépend des caractéristiques de l'opérateur (Tableau 4). Nous nous concentrons sur les réseaux et les périodes associées à l'arrivée de nouveaux régulateurs (conseils municipaux), ce qui nous permet de construire un échantillon de 53 séries de deux périodes contractuelles.

\subsection{Distribution des paramètres d'efficacité des opérateurs de transport}

Nous estimons la distribution du paramètre d'efficacité $\theta$ des opérateurs dans les deux scénarios: Celui plus probable où l'autorité utilise des contrats robustes à la renégociation et augmente les subventions d'une période à l'autre, et celui moins crédible où il n'y a pas d'apprentissage de la part de l'autorité qui se limite alors à reconduire le même contrat d'une période à l'autre.

Contrats avec engagement: Si l'on observe une situation où l'opérateur n'accepte pas un contrat de type «prix-fixe», c'est parce que cet opérateur fait face à un coût $\theta$ important qui l'empêche d'obtenir des bénéfices positifs. En faisant coïncider la distribution de $\theta$ à la probabilité empirique d'accepter un contrat prix fixe, nous sommes capables de récupérer cette distribution.

\footnotetext{
la base de données montre que, sur une période contractuelle, plusieurs objectifs peuvent être affectés par de légères fluctuations. Ce peut être par exemple le cas de l'offre de transport, mesurée par la quantité de places-kilomètres disponibles, qui affectera à son tour les coûts et les volumes des subventions. Ces fluctuations sont dues à des chocs exogènes qui peuvent affecter l'activité de l'opérateur au cours de la période contractuelle: Modification des conditions du trafic, ou de la configuration du réseau, routes en travaux, ou grèves sont des exemples potentiels. Les réponses économiques à ces chocs prévisibles sont anticipées par le contrat. De là, bien que les objectifs du contrat puissent fluctuer en cours de période contractuelle, ils sont les éléments d'un seul et même contrat. Au lieu de calculer une simple moyenne pour chaque objectif sur la période contractuelle lorsque des fluctuations sont présentes, nous choisissons de traiter chaque fluctuation comme une donnée indépendante afin d'augmenter les degrés de liberté de l'analyse.
} 
L'opérateur accepte un contrat prix fixe lorsque (2) est satisfaite. La probabilité d'accepter un contrat prix fixe est donc

$$
\operatorname{Pr}\left(\theta \leq b_{F}+k\right)=F\left(b_{F}+k, \mu_{G}, \sigma_{G}^{2}\right),
$$

où $F($.$) est la fonction de distribution avec densité f\left(., \mu_{G}, \sigma_{G}^{2}\right)$. Nous supposons que les $\theta$ s sont tirés indépendamment d'une distribution normale qui est commune à tous les réseaux de transport et tous les opérateurs.

Le terme de droite de l'équation (9) est la distribution que nous souhaitons estimer. En particulier, il est nécessaire d'évaluer la moyenne $\mu_{G}$ et la variance $\sigma_{G}^{2}$ de la distribution normale. Comme ces paramètres nous sont inconnus, nous devons utiliser une spécification alternative de la probabilité du choix de contrat prix fixe. Nous supposons donc que cette probabilité peut être approchée par une spécification logit où la probabilité de choisir un contrat de type «prix fixe» dépend de plusieurs variables explicatives. ${ }^{20}$ De là, nous faisons coïncider la probabilité empirique logit de choisir un contrat «prix fixe» avec la fonction de distribution évaluée au point $b_{F}+k$ :

$$
\operatorname{Pr}(P / S)=\operatorname{Pr}\left(\theta \leq b_{F}+k\right)=F\left(b_{F}+k, \mu_{G}, \sigma_{G}^{2}\right),
$$

où $P$ signifie prix fixe et $S=\{P, C\}$. Dans un second temps, nous récupérons les paramètres $\mu_{G}$ et $\sigma_{G}^{2}$ :

$$
\min _{\left\{\mu_{G}, \sigma_{G}^{2}\right\}} \sum_{i}\left[\operatorname{Pr}(P / S)-F\left(b_{F}+k, \mu_{G}, \sigma_{G}^{2}\right)\right]^{2} .
$$

Nous supposons que $k$ est constant, et réalisons la normalisation suivante: A partir de (9), nous réécrivons:

$$
\operatorname{Pr}(P / S)=\operatorname{Pr}\left(\theta^{\prime}=\theta-k \leq b_{F}\right)=F\left(b_{F}, \mu_{G}^{\prime}, \sigma_{G}^{2}\right) .
$$

De là, nous évaluons la moyenne $\mu_{G}^{\prime}$ et la variance $\sigma_{G}^{2}$ de la distribution de $\theta^{\prime}$. Une estimation de $\mu_{G}$ est simplement $\mu_{G}=\mu_{G}^{\prime}+k$.

Nous déterminons maintenant la fonction logit de choix de contrats prix fixe. L'opérateur sélectionne le contrat $s$ qui lui donne le niveau d'utilité le plus élevé. La probabilité de choix du contrat $s$ est égale à la probabilité que l'utilité de l'alternative $S=\{P, C\}$ soit supérieure ou égale à l'utilité de l'autre alternative:

\footnotetext{
${ }^{20}$ Cette procédure est similaire à celle utilisée par Ryan [2006] pour représenter les choix d'entrée des producteurs de ciment à l'intérieur de marchés locaux aux Etats-Unis. Voir aussi Bajari et alii. [2007] et Hotz et Miller [1993].
} 


$$
\operatorname{Pr}(s / S)=\operatorname{Pr}\left(U_{i}^{s} \geq U_{i}^{s^{\prime}}\right), \quad s, s^{\prime} \in\{P, C\} .
$$

Nous pouvons exprimer l'utilité aléatoire d'une alternative comme la somme des composantes observables et inobservables de l'utilité totale:

$$
U_{i}^{s}=\bar{U}^{s}\left(x_{i}\right)+\omega^{s}\left(x_{i}\right)=\bar{U}_{i}^{s}+\omega_{i}^{s},
$$

où $x_{i}^{s}$ est un vecteur de caractéristiques de l'opérateur qui varie d'un individu à l'autre. Nous pouvons donc réécrire notre probabilité ainsi:

$$
\operatorname{Pr}(s / S)=\operatorname{Pr}\left(\omega_{i}=\omega_{i}^{s^{\prime}}-\omega_{i}^{s} \leq \bar{U}_{i}^{s}-\bar{U}_{i}^{s^{\prime}}\right), \quad s, s^{\prime} \in\{P, C\} .
$$

Si l'erreur $\omega_{i}$ suit une distribution logistique:

$$
\operatorname{Pr}(s / S)=\frac{\exp \left(\bar{U}_{i}^{s}\right)}{\exp \left(\bar{U}_{i}^{s}\right)+\exp \left(\bar{U}_{i}^{s^{\prime}}\right)}, \quad s, s^{\prime} \in\{P, C\} .
$$

\subsubsection{Contrats robustes à la renégociation}

Pour récupérer la distribution des $\theta$ s, nous répliquons la méthodologie présentée ci-dessus, en l'appliquant au cas particulier des contrats robustes à la renégociation. Trois types d'arrangements contractuels sont maintenant considérés (au lieu de deux précédemment): Rappelons que nous expliquons maintenant non pas simplement un choix de contrat mais plutôt un choix de série de contrats réalisé par l'opérateur au début d'une série de deux périodes contractuelles: Une série de contrats prix fixes $(P P)$, une série de contrats coûts du service $(C C)$, ou un contrat coûts du service suivi d'un contrat prix fixe $(C P)$. Si un opérateur n'accepte pas un contrat prix fixe (à aucune des périodes), c'est sans doute parce qu'il a un type $\theta$ très fort. De la même manière, si l'opérateur accepte un contrat prix fixe au cours des deux périodes (de la seconde période seulement resp.), c'est parce que le paramètre $\theta$ est faible (moyen resp.). ${ }^{21}$

L'opérateur accepte un contrat prix fixe au cours des deux périodes lorsque:

$$
\theta \leq \theta_{1}^{*}=b_{1}+k .
$$

\footnotetext{
${ }^{21}$ Une quatrième possibilité est la passage d'un contrat prix fixe à un contrat coût du service. Très peu d'arrangements contractuels de ce type sont observés dans notre base de données. Nous choisissons de ne pas considérer les rares réseaux concernés par ces changements.
} 
La probabilité d'accepter un contrat prix fixe sur deux périodes est la probabilité de bénéficier d'un niveau d'inefficacité $\theta$ inférieur à $b_{1}+k$ :

$$
\operatorname{Pr}\left(\theta \leq b_{1}+k\right)=F\left(b_{1}+k, \mu_{R P}, \sigma_{R P}^{2}\right),
$$

où $F($.$) est la fonction de distribution avec densité f\left(., \mu_{R P}, \sigma_{R P}^{2}\right)$. Nous supposons à nouveau que les $\theta$ s sont distribués normalement.

L'opérateur accepte un contrat prix fixe sur deux période ou sur la seconde période seulement si

$$
\theta \leq \theta_{2}^{*}=b_{2}+k
$$

La probabilité d'accepter un de ces deux arrangements contractuels est la probabilité d'obtenir un tirage sur $\theta$ inférieur à $b_{2}+k$ :

$$
\operatorname{Pr}\left(\theta \leq b_{2}+k\right)=F\left(b_{2}+k, \mu_{R P}, \sigma_{R P}^{2}\right) .
$$

Le membre de droite des équations (13) et (14) est la distribution que nous souhaitons estimer. Les deux paramètres $\mu_{R P}$ et $\sigma_{R P}^{2}$ sont inconnus et doivent donc être évalués.

Nous supposons maintenant que la probabilité d'observer un de ces trois arrangements contractuels peut être approchée par une spécification multinomial logit. Nous estimons ces probabilités dans un premier temps. Ensuite, nous faisons coïncider la probabilité empirique de choisir un des trois régimes contractuels, telle que définie par la spécification multinomial logit, avec la fonction de distribution évaluée aux points $b_{1}+k$ et $b_{2}+k$ :

$$
\operatorname{Pr}(P P / \Gamma)=\operatorname{Pr}\left(\theta \leq b_{1}+k\right)=F\left(b_{1}+k, \mu_{R P}, \sigma_{R P}^{2}\right)
$$

et

$$
\operatorname{Pr}(C P / \Gamma)+\operatorname{Pr}(P P / \Gamma)=\operatorname{Pr}\left(\theta \leq b_{2}+k\right)=F\left(b_{2}+k, \mu_{R P}, \sigma_{R P}^{2}\right),
$$

où $\Gamma=\{P P, C C, C P\}$. Pour récupérer les paramètres $\mu_{R P}$ et $\sigma_{R P}^{2}$, nous résolvons le système:

$\min _{\left\{\mu_{R P}, \sigma_{R P}^{2}\right\}} \sum_{i}\left[\operatorname{Pr}(P P)-F\left(b_{1}+k, \mu_{R P}, \sigma_{R P}^{2}\right)\right]^{2} \sum_{i}\left[\operatorname{Pr}(C P)+\operatorname{Pr}(P P / \Gamma)-F\left(b_{2}+k, \mu_{R P}, \sigma_{R P}^{2}\right)\right]^{2}$.

Nous évaluons la moyenne $\mu_{R P}^{\prime}$ et la variance $\sigma_{R P}^{2}$ de la distribution de $\theta^{\prime}=\theta-k$. Une estimation de $\mu_{R P}$ est $\mu_{R P}=\mu_{R P}^{\prime}+k$. 
Nous procédons maintenant à la définition de notre spécification multinomial logit. Si l'opérateur choisit l'arrangement contractuel $r \in\{P P, C P, C C\}$, nous supposons que $U_{i}^{r}$ est maximale parmi les $R$ arrangements.

La probabilité que le choix $r$ est réalisé est

$$
\operatorname{Pr}\left(U_{i}^{r} \geq U_{i}^{r^{\prime}}\right), \text { pour } r^{\prime} \neq r
$$

Comme dans le cas précédent, les équations estimées fournissent un ensemble de probabilités pour les $R$ choix d'un opérateur de caractéristiques $x_{i}$. Les probabilités sont:

$$
\operatorname{Pr}(r / R)=\frac{\exp \left(\bar{U}_{i}^{r}\right)}{1+\sum_{k=1}^{R-1} \exp \left(\bar{U}_{i}^{k}\right)}, \quad r \in\{C P, C C\}
$$

et

$$
\operatorname{Pr}(r=P P)=\frac{1}{1+\sum_{k=1}^{R-1} \exp \left(\bar{U}_{i}^{k}\right)} .
$$

\subsection{Résultats}

Nous présentons maintenant les résultats d'estimation obtenus dans chacun des deux scenarios définis ci-dessus.

Contrats avec engagement. Le tableau 2 présente les estimations du modèle logit de choix de contrats prix fixe présenté dans l'équation (12). Les variables explicatives sont: Une constante, la taille totale du réseau, le pourcentage d'ingénieurs dans la force de travail totale, le volume de subvention par unité d'offre, une variable muette égale à 1 si l'opérateur appartient au group Keolis, et 0 sinon, une variable muette égale à 1 si l'opérateur appartient au group Connex, et 0 sinon, et une variable muette égale à 1 si l'opérateur appartient au group Agir, et 0 sinon. ${ }^{22}$

Nous présentons quatre types d'estimations. Les paramètres obtenus sont en majorité très significatifs. Notons que la probabilité de choisir un contrat «prix fixe» augmente avec la taille du réseau. Ce résultat illustre sans doute le fait que les opérateurs de taille supérieure bénéficient d'une expérience plus élevée, et que les économies d'échelle sont

\footnotetext{
${ }^{22}$ Les régies de transport concernent les réseaux où l'autorité organisatrice opère directement le service. C'est le cas principalement des grandes municipalités comme Paris, Marseille, ou Lyon. Nous nous intéressons uniquement aux réseaux où l'autorité organisatrice délègue le service à un opérateur et établit un contrat.
} 
plus importantes. Un tel effet taille nous aide à identifier le paramètre d'efficacité $\theta$ de l'opérateur.

Un autre résultat intéressant est l'effet positif de la concentration des ingénieurs dans la force de travail totale de l'entreprise. Notons toutefois que l'effet n'est pas significatif. Cette variable fournit une mesure de la dotation en capacité productive de chaque entreprise. Les ingénieurs sont généralement responsables des activités de recherche et développement dans le contrôle de la qualité, la maintenance et l'efficacité productive. Leur action est particulièrement importante pour l'amélioration de la vitesse moyenne du réseau. Une plus grande dotation accroît la probabilité de choisir un contrat prix fixe. La variable subvention par unité d'offre a un effet positif sur la probabilité de choix d'un contrat prix fixe. Ce résultat est logique et confirme l'intuition de notre modèle théorique: Une plus grande subvention garantie une meilleure couverture des coûts espérés, et augmente ainsi la probabilité d'acceptation d'un contrat incitatif. Enfin, les trois variables muettes qui rendent compte de l'identité du groupe auquel appartient l'opérateur ${ }^{23}$ sont significatives. Ceci suggère que ces groupes ont des préférences significatives pour un certain type de contrat.

A partir de l'estimation logit du choix de contrat (modèle IV), nous pouvons récupérer les probabilités $\operatorname{Pr}(s / S)$ nécessaires à l'évaluation de la distribution $F\left(\theta, \mu_{G}, \sigma_{G}^{2}\right)$. Le Tableau 3 présente les estimations de $\mu_{G}^{\prime}$ et $\sigma_{G}$, qui sont respectivement la moyenne et l'écart type de la distribution de $\theta^{\prime}$. Une estimation de $\mu_{G}$ est $\mu_{G}=\mu_{G}^{\prime}+k$.

Contrats robustes à la renégociation. Les résultats d'estimation du modèle multinomial logit sont présentés dans le Tableau 4. L'arrangement contractuel $P P$ est le choix de référence. De là, les coefficients estimés reflètent les effets des $x_{i}$ sur la probabilité de choisir $r$ relative au choix de l'arrangement $P P$. Les variables explicatives sont les mêmes que celles du modèle logit ci-dessus. Une fois encore, le modèle s'adapte très bien aux données.

On note tout d'abord que la taille du réseau (et donc la taille de l'opérateur) a un effet significatif sur le choix du contrat. Une fois encore, les contrats à prix fixe (dans les arrangements $P P$ ou $C P$ ) son préférés par les opérateurs plus importants en taille. Ensuite, le volume de la subvention versée à l'opérateur joue un rôle important: L'entreprise est plutôt disposée à choisir une série de contrats à «prix fixes» si la subvention par unité d'output est plus élevée. De la même manière, l'entreprise choisit la combinaison «coût du

\footnotetext{
${ }^{23}$ Ici, le groupe de référence est Connex.
} 
service» puis «prix fixe» plutôt qu'une série de contrats «coût du service» si la subvention unitaire est plus élevée.

La part des ingénieurs produit également le résultat attendu. Une plus grande dotation en capacité productive accroît la probabilité de choix du contrat «prix fixe». L'effet est plus important dans le cas d'une série de contrats prix fixe comparé à une situation où un contrat prix fixe succède à un contrat coût du service. Finalement, on observe un effet groupe important. En particulier, Transdev ${ }^{24}$ est caractérisé par une préférence plus forte pour les séries de contrats «prix fixes» que les autres groupes.

Comme dans le cas précédent, nous pouvons récupérer à partir de la spécification multinomial logit (Modèle I) les probabilités $\operatorname{Pr}(r / R)$ afin d'évaluer la distribution $F\left(\theta, \mu_{R G}, \sigma_{R G}^{2}\right)$ de $\theta$ dans un contexte de régimes contractuels robustes à la renégociation. Comme $k$ est inconnu, Nous évaluons la moyenne $\mu_{R P}^{\prime}$ et la variance $\sigma_{R P}^{2}$ de la distribution de $\theta^{\prime}=\theta-k$. Les résultats sont fournis dans le Tableau 5. Une estimation de $\mu_{R P}$ est $\mu_{R P}=\mu_{R P}+k$.

Contrats avec engagement contre contrats robustes à la renégociation. On remarque que le scénario réglementation robuste à la renégociation implique une moyenne supérieure à la moyenne obtenue sous l'hypothèse de réglementation avec engagement. Ainsi, une mauvaise hypothèse quant au comportement des autorités de transport conduit à un biais au moment de l'estimation de la distribution du paramètre d'efficacité $\theta$ des opérateurs de transport. Comme nous l'avons suggéré dans cet article, l'utilisation de contrats robustes à la renégociation est l'hypothèse la plus pertinente. Supposer au contraire que ces autorités s'engagent à ne pas modifier les termes des contrats de transport conduirait donc à l'impression erronée que les opérateurs sont plus efficaces qu'ils ne le sont en réalité.

\section{References}

bajari p., benkard l, levin j.[2007], «Estimating Dynamic Models of Imperfect Competition», Econometrica, 75, p. 1331-1370.

baron d., myerson r. [1982], «Regulating a Monopolist with Unknown Costs», Econometrica, 50, p. 911-930.

gagnepain p., ivaldi m., martimort d. [2008], «The Cost of Contract Renegotiation For Public Transport Services», Mimeo IDEI.

\footnotetext{
${ }^{24}$ Ici, le groupe de référence est Connex.
} 
gasmi f., laffont j.j., sharkey w. [1995], «Incentive Regulation and the Cost Structure of the Local Telephone Exchange Network», Journal of Regulatory Economics, 12, p. 5-25.

hotz v., miller r. [1994], «Conditional Choice Probabilities and the Estimation of Dynamic Models», Review of Economic Studies, 60, p. 497-529.

institut d'économie industrielle [1999], «Network Industries and Public Service», European Commission Reports and Studies, 4.

laffont j.j., martimort d. [2002], The Theory of Incentives: The PrincipalAgent Model, Princeton: Princeton University Press.

laffont j.j., tirole j. [1993], A Theory of Incentives in Regulation and Procurement, Cambridge: MIT Press.

rogerson w. [2003], «Simple Menus of Contracts in Cost-Based Procurement and Regulation », American Economic Review, 93, p. 919-926.

ryan s. [2006], «The Cost of Environmental Regulation in a Concentrated Industry», Mimeo MIT.

wilson r. [1993], Nonlinear Pricing, Oxford: Oxford University Press.

wolak f. [1994], «An Econometric Analysis of the Asymmetric Information Regulator-Utility Interaction» Annales d'Economie et de Statistiques, 34, p. $13-69$.

wunsch p. [2004], «Estimating Menus of Linear Contracts for Mass Transit Firms», Mimeo CORE. 


\begin{tabular}{lcr}
\hline Variables & Moyenne & Ecart type \\
\hline Coûts (Milliers Euros) & 10,940 & $19,273,852$ \\
Subvention (Milliers Euros) & 11,093 & 9,597 \\
Sub / unité d'offre (Euro) & 0.016 & 0.005 \\
Taille du réseau (km) & 288.3 & 200.1 \\
Part des ingénieurs & 0.293 & \\
Part gouvernements de droite & 0.526 & \\
Part opérateurs publics & 0.387 & \\
Part contrats prix fixe & 0.555 & \\
Part Keolis & 0.326 & \\
Part Agir & 0.163 & \\
Part Connex & 0.224 & \\
Part Transdev & 0.245 & \\
\hline
\end{tabular}

Table 1: Données

Figure 1: 


\begin{tabular}{lccll}
\hline Variables & I & II & III & IV \\
\hline Constante & $-4.73^{* * *}$ & $5.09^{* * *}$ & $5.14^{* * *}$ & $7.41^{* * *}$ \\
Taille du réseau & $(0.75)$ & $(1.44)$ & $(1.46)$ & $(1.65)$ \\
& $0.91^{* * *}$ & $0.88^{* * *}$ & $0.82^{* * *}$ & $0.77^{* * *}$ \\
Sub / unité d'offre & $(0.13)$ & $(0.14)$ & $(0.15)$ & $(0.15)$ \\
& & $2.22^{* * *}$ & $2.26^{* * *}$ & $2.68^{* * *}$ \\
Part ingénieurs & & $(0.29)$ & $(0.30)$ & $(0.35)$ \\
Agir & & & 1.31 & \\
Keolis & & & & $-0.58^{* * *}$ \\
& & & & $(0.27)$ \\
Transdev & & & & -0.21 \\
& & & & $(0.21)$ \\
Moyenne Log Vraisemblance & -0.65 & -0.60 & -0.60 & -0.54 \\
\# Contrats & & & & \\
\hline
\end{tabular}

Table 2: Choix de contrats prix fixes avec engagement total

\begin{tabular}{lcr}
\hline Distribution de $\theta^{\prime}$ & Estimation & Ecart type \\
\hline Moyenne $\mu_{G}^{\prime}$ & $13,821^{* * *}$ & 1,835 \\
Ecart type $\sigma_{G}$ & $15,240^{* * *}$ & 3,933 \\
\hline
\end{tabular}

Table 3: Distribution estimée avec engagement total (Valeurs en milliers d'Euros) 


\begin{tabular}{lcccc}
\hline \multirow{2}{*}{ Variables } & \multicolumn{2}{c}{ I } & \multicolumn{2}{c}{ II } \\
\hline & Paramètres & Ecart type & Paramètres & Ecart type \\
Série CC & & & & \\
Constante & & & & \\
Taille du réseau & $-7.63^{* * *}$ & 2.24 & $-4.56^{* *}$ & 1.79 \\
Sub / unité d'offre & $-0.81^{* * *}$ & 0.22 & $-1.08^{* * *}$ & 0.19 \\
Part ingénieurs & $-2.75^{* * *}$ & 0.44 & $-2.21^{* * *}$ & 0.34 \\
Agir & & & $-0.82^{* * *}$ & 1.19 \\
Keolis & $0.88^{* * *}$ & 0.32 & & \\
Transdev & 0.06 & 0.28 & & \\
Série CP & $-3.40^{* * *}$ & 0.51 & & \\
Constante & & & & \\
Taille du réseau & & & & \\
Sub / unité d'offre & & & & \\
Part ingénieurs & $-13.96^{* * *}$ & 2.09 & $-10.26^{* * *}$ & \\
Agir & $0.60^{* * *}$ & 0.18 & $0.41^{* *}$ & \\
Keolis & $-2.55^{* * *}$ & 0.41 & $-1.71^{* * *}$ & 0.33 \\
Transdev & & & $-0.61^{* * *}$ & 0.86 \\
Moyenne Log Vraisemblance & $-1.05^{* * *}$ & 0.36 & & \\
\# Series de Contrats & -0.05 & 0.20 & & \\
\hline
\end{tabular}

Table 4: Choix d'une série de contrats CC ou CP avec robustesse à la renégociation

\begin{tabular}{lcr}
\hline Distribution de $\theta^{\prime}$ & Estimation & Ecart type \\
\hline Moyenne $\mu_{G}^{\prime}$ & $16,926^{* * *}$ & 2,433 \\
Ecart type $\sigma_{G}$ & $17,508^{* * *}$ & 4,392 \\
\hline
\end{tabular}

Table 5: Distibution estimée avec robustesse à la renégociation (Valeurs en milliers d'Euros) 